\title{
Netzwerkbörse für Qualitätsfachleute
}

\author{
Interview: Fabienne Hohl
}

Möchten Sie der Fachwelt ein innovatives und praxisbewährtes Qualitätsprojekt vorstellen? Der Swiss Quality Award 2016 bietet Ihnen eine ideale Plattform dafür. Wie sich der Preis für das Réseau Douleur, Gewinner des Jahres 2013, ausgewirkt hat, berichtet Dr. med. Christophe Luthy von den Hôpitaux Universitaires Genève im Interview.*

\begin{abstract}
Ihr Team des Réseau Douleur hat 2013 mit seinem Projekt "Agissons ensemble contre la douleur!» zur interdisziplinären Verbesserung der Schmerzbehandlung im stationären Bereich den Swiss Quality Award gewonnen. Wodurch hat sich dieses Projekt besonders ausgezeichnet?

Diese Frage können die Jurymitglieder des Swiss Quality Award sicher viel besser beantworten! Das Team des Schmerznetzwerks möchte seine Begeisterung und Kreativität auf die interdisziplinär tätigen Mitarbeiterinnen und Mitarbeiter des Genfer Universitätsspitals (HUG) übertragen. Ich hoffe, dass diese Elemente als entscheidende Faktoren unseres Qualitätsansatzes beurteilt wurden. Die Schmerzbehandlung zu verbessern ist ein langwieriger Prozess. Uns geht es dabei insbesondere darum, den Gedankenaustausch zu fördern und dafür zu sorgen, dass Kompetenzen auf verschiedenen Ebenen stärker gemeinsam genutzt werden. An einer Institution wie den HUG (Hôpitaux Universitaires Genève) gibt es verschiedenste Ressourcen, z.B. technische Hilfsmittel, aber vor allem auch motiviertes und gut ausgebildetes Personal. Zwischen all diesen Ressourcen gilt es, Synergien zu schaffen. Erstere gehören umfassender genutzt, um kranke Menschen optimal zu betreuen.
\end{abstract}

Was hat Sie dazu bewogen, sich mit Ihrem Projekt
gerade für den Swiss Quality Award zu bewerben? Ein massgebendes Element war für uns die Präsenz in der Öffentlichkeit, die mit dieser Plattform verbunden ist. Diese gibt uns die Möglichkeit, unsere Aktivitäten bekannt zu machen und Kontakte mit anderen Kolleginnen und Kollegen zu knüpfen. Im Auge hatten wir dabei sowohl die lokale Ebene als auch Ansprechpartner in der ganzen Schweiz und im Ausland.

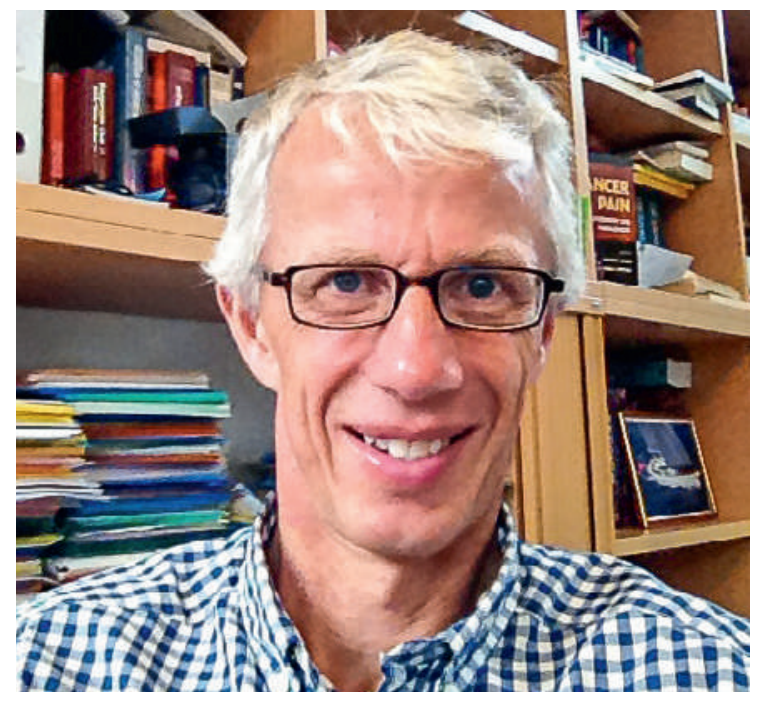

Dr. med. Christophe Luthy, Präsident des Réseau Douleur an den Hôpitaux Universitaires Genève: «Der Swiss Quality Award ermöglichte es uns, viele weitere lokale, nationale und internationale Kontakte zu knüpfen.»

\section{Und was hat der Award dem Schmerznetzwerk gebracht?}

In den letzten Jahren haben wir unsere interdisziplinäre Zusammenarbeit sowohl innerhalb des HUG als auch mit externen Partnern ausgebaut. Zu den Letzteren gehören Berufsfachschulen, Spitex-Organisationen und andere Spitäler, insbesondere in den Kantonen Waadt, Neuenburg und Wallis. Ausserdem wurden wir von der Schweizerischen Gesellschaft zum Studium des Schmerzes (SGSS/SSED) mit einem ersten Preis ausgezeichnet.

Was gibt es seit Verleihung des Swiss Quality Award 2013 Neues beim Schmerznetzwerk?

Das Schmerznetzwerk wird kontinuierlich weiterentwickelt (www.hug-ge.ch/reseau-douleur). Kurz gesagt 
wird der Schmerz heute noch stärker als Indikator für die Versorgungsqualität herangezogen. Dies erfolgt hauptsächlich über die Ausbildung und über Umfragen, die wir unter den multidisziplinär tätigen Mitarbeitenden und den Patientinnen und Patienten durchführen. Des Weiteren erarbeiten und verteilen wir Informationsmaterial und Entscheidungshilfen. In den letzten 18 Monaten ging es uns vor allem darum, das Management von behandlungsbedingten Schmerzen zu verbessern, sprich die klinische Praxis und deren Dokumentation in den Krankengeschichten. Zudem arbeiten wir daran, die postoperative Schmerztherapie zu optimieren, indem wir Fachpersonen ausbilden, berufsübergreifend koordinieren und laufend neue Empfehlungen für die Institutionen herausgeben.

\section{Wo würden Sie in der Versorgungsqualität die Prioritäten setzen?}

Die heutige Gesundheitsversorgung befindet sich auf mehreren Ebenen im Wandel. Dazu gehören etwa die Möglichkeiten der Medizin, die Ansprüche und das Profil der Patientinnen und Patienten, die Interdisziplinarität, die Koordination zwischen den Spitälern und der ambulanten Versorgung, die Art der Vergütung sowie die Budgetkontrolle. Persönlich lege ich grossen Wert auf die Verbindungen zwischen der Gesundheitsversorgung und der Forschung, damit sich die theoretischen Kenntnisse in die Praxis übertragen lassen und die Forschung einen unmittelbaren Bezug zur Praxis hat. Diese gegenseitige Öffnung und die Zusammenarbeit sind eine unabdingbare Voraussetzung, um Fortschritte im Bereich der Versorgungsqualität zu erzielen und ein gutes Kostenmanagement zu gewährleisten. Konkrete Beispiele dafür sind Projekte, welche die Partnerschaft mit dem Patienten und die Patientenzufriedenheit untersuchen. Oder Aktivitäten, welche

\section{Gewinnen Sie den Swiss Quality}

Award 2016!

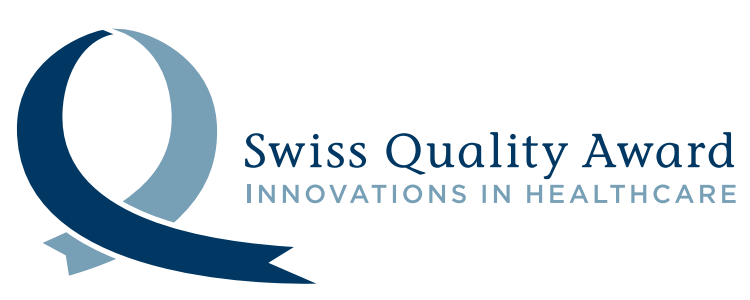

Qualitätsinnovationen im Gesundheitswesen verdienen Bekanntheit - diese zu schaffen, Pioniere zu vernetzen und praxisrelevante Erkenntnisse verfügbar zu machen sind die Ziele des Swiss Quality Award. Der Preis prämiert überzeugende, praxiserprobte Qualitätsprojekte in den Kategorien ambulanter Sektor, stationärer Sektor und sektorübergreifend mit je 10000 Franken. Überdies vergibt die Jury unter den besten Qualitätsarbeiten einen Posterpreis von 2000 Franken. Melden Sie Ihr Projekt heute an! Anmeldeschluss ist der 8. Januar 2016. Weitere Informationen finden Sie auf www.swissqualityaward.ch

die interdisziplinäre Organisation der Gesundheitsversorgung optimieren wollen. Solchen Anstrengungen muss meiner Auffassung nach Priorität eingeräumt werden. Meine beiden Schlüsselwörter lauten daher «Partnerschaft mit den Patientinnen und Patienten» sowie «Interdisziplinarität»! Denn für die heutige Medizin ist ein intensiver Informationsaustausch erforderlich, um nutzenstiftende Absprachen und fruchtbare Partnerschaft zu erreichen. Im Zusammenhang mit komplexen Behandlungen gilt es sowohl klinische als auch organisatorische Aspekte zu beachten. Wenn Ärztinnen und Ärzte besser mit den anderen Gesundheitsfachpersonen zusammenarbeiten, wie auch der ambulante stärker mit dem stationären Sektor, wird sich dies sehr positiv auf die Versorgungsqualität auswirken.

\section{In Gedenken an Prof. Dr. med. Christoph Röder}

Am 23. August 2015 ist Christoph Röder unerwartet verstorben. Der plötzliche Abschied macht uns alle betroffen und hinterlässt eine grosse Lücke. Christoph Röder leitete das Institut für Evaluative Forschung in der Medizin (IEFM) der Universität Bern. Dieses vertrat er sowohl beim "Swiss Quality Award" als auch beim «Nationalen Symposium für Qualitätsmanagement». Mit seinem Engagement und Enthusiasmus, seinen innovativen Inputs, seiner kollegialen und humorvollen Art prägte er den Swiss Quality Award und das Symposium entscheidend und gestaltete die medizinische Qualitätssicherungslandschaft in der Schweiz mit. Sein gutes Netzwerk ermöglichte immer wieder fruchtbare Kooperationen und viele spannende Referierende am Symposium. Wir denken mit grosser Dankbarkeit und tiefer Trauer an Chris Röder. Wir vermissen ihn.

Die Trägerorganisationen des Swiss Quality Award Schweiz. Gesellschaft für Qualitätsmanagement im Gesundheitswesen Institut für Evaluative Forschung in der Medizin der Universität Bern FMH, Departement Daten, Demographie und Qualität / Schweizerische Akademie für Qualität in der Medizin 